\title{
FIREX project and effects of self-generated electric and magnetic fields on electron-driven fast ignition
}

\author{
Kunioki Mima , A Sunahara , Hiroyuki Shiraga , H Nishimura , \\ H Azechi , T Nakamura , T Johzaki , H Nagatomo , C Garcia and \\ $P$ Veralde \\ Institute of Laser Engineering, Osaka University, 2-6 Yamadaoka, Suita, Osaka, \\ 565-0871, Japan \\ Graduate School for the Creation of New Photonics Industries, 1955-1, Kurematsu, Nishiku, \\ Hamamatsu, Shizuoka, 431-1202, Japan \\ Institute of Nuclear Fusion, University Politecnica de Madrid, C/José Gutiérrez Abascal, \\ 2, 28006 Madrid, Spain
}

\begin{abstract}
Fast ignition is a new scheme in laser fusion, in which higher energy gain with a smaller laser pulse energy is expected. A cone target has been introduced for realizing higher coupling efficiency. At ILE, Osaka University, a laser with four beams and a total output of $10 \mathrm{~kJ} \mathrm{ps}^{-1}$, laser for fast ignition experiment (LFEX), has been constructed and we have carried out an integrated experiment with one beam of the LFEX. Through experiments it was found that the coupling efficiency is degraded when the laser pre-pulse is not sufficiently small. Namely, the main pulse is absorbed in the long-scale pre-plasma produced by the pre-pulse and the hot electron energy is higher than that for a clean pulse. Furthermore, the distance between the hot electron source and the core plasma is large. Hence, we are exploring how to overcome the pre-pulse effects on the cone target.

In this paper it is proposed that a thin foil covers the laser entrance of the cone to mitigate the pre-plasma and a double cone reduces the loss of highenergy electrons from the side wall of the cone. The simulations indicate that a higher coupling efficiency is expected for the double cone target with a thin foil at the laser entrance. Namely, the pre-pulse will be absorbed by the foil and the electromagnetic fields generated on the surface of the inner cone will confine high-energy electrons.
\end{abstract}

(Some figures in this article are in colour only in the electronic version)

\section{Introduction}

By PW laser experiments, the imploded plasma has been heated up to about $1 \mathrm{keV} \mathrm{[1]} \mathrm{in} \mathrm{the} \mathrm{Joint}$ experiments of Osaka University and Rutherford Appleton Laboratory to clarify the heating 


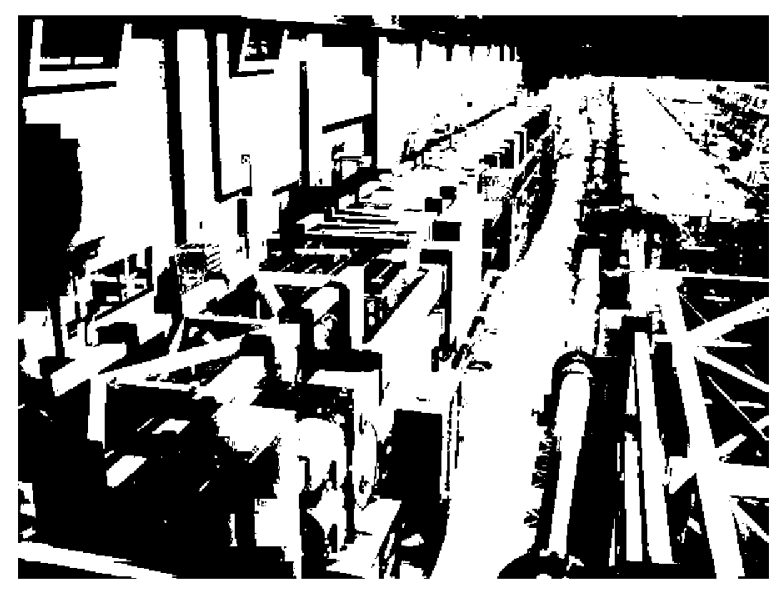

Figure 1. $10 \mathrm{~kJ} / 10 \mathrm{ps}$ LFEX laser.

scaling law in the relations between heating laser energy, hot spark temperature and neutron yield. Using the Fokker-Planck simulations, cone shell target experiments were analyzed. Relativistic electrons are generated more efficiently and the spectrum is predicted to be double Maxwellian. Then, the ion temperature will reach above $1 \mathrm{keV}$. The results can be extended to the FIREX. Namely, the coupling efficiency is higher than $10 \%$ and the required heating laser energy for achieving $5 \mathrm{keV}$ (the goal of Fast Ignition Realization Experiment I; FIREX-I) is estimated to be $10 \mathrm{~kJ}$ for 100 times solid density DT plasmas with $30 \mu \mathrm{m}$ diameter [2]. For fast. ignition, the critical parameters are the hot spot radius, $r_{b}$, and the coupling efficiency which strongly depend upon the relativistic electron spectrum, source stand-off distance and transport. According to the simulation and previous experiment results, the relativistic electron heat flow can be confined by self-generated magnetic fields in the cone shell target. Then, the deposited laser energy is concentrated on the top of the cone and the hot spark radius could be controlled by the cone tip radius and the position of the cone tip. From the present understanding of the heating processes, the ignition can be achieved with a pulse energy less than $50 \mathrm{~kJ} / 10 \mathrm{ps}$ for an imploded plasma of $\rho R$ above $1.0 \mathrm{~g} \mathrm{~cm}^{-2}[2,3]$.

For demonstrating the feasibility of fast ignition, the LFEX laser has been completed at Osaka University (Laser for Fast ignition Experiment: LFEX), figure 1. The LFEX is coupled with the Gekko XII laser of $10 \mathrm{~kJ} \mathrm{~ns}^{-1}$ with $0.53 \mu \mathrm{m}$. The one-beam experiment with the LFEX has started. However, the preliminary experiments indicate that the enhancement of neutron yield is not as high as it was expected because of the laser pre-pulse. So, toward the FIREX-I, an advanced target is proposed for achieving a better heating performance. The designed target is shown in figure 2. Here, we have introduced a thin foil at the laser entrance of the cone, a double cone wall, a low- $Z$ (plastic) coating for the inner and outer surfaces, which leads to a higher implosion $\rho R$ (as indicated by Nagatomo et al [4]), and a Br-doped ablator for the fuel shell for stabilizing the $R-T$ instability.

In this paper, we will summarize the results of the preliminary experiments and discuss the physics related to the advanced target.

\section{Summary of the preliminary results of the FIREX-I experiments}

The $10 \mathrm{~kJ} \mathrm{ps}^{-1}$ short pulse laser LFEX, for fast ignition, was dedicated on March 2009. The heating experiments started in June 2009. One beam of the heating laser was injected 


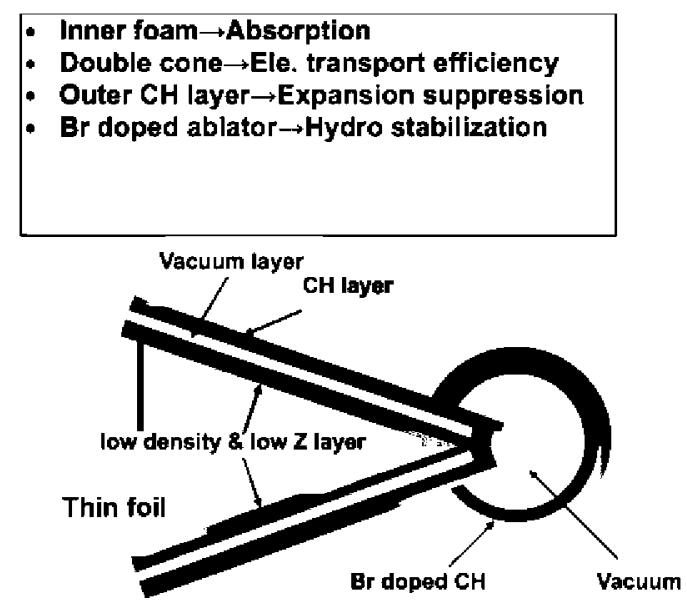

Figure 2. Advanced target design for FIREX-I [3, 5].

into the cone. The shot timing was measured by an x-ray streak camera. The results indicate that the LFEX x-ray signal and the x-ray from the imploded core are coincident. Namely, a petawatt laser pulse could be synchronized with the maximum compression timing. In this case, the neutron yield is enhanced. The plasma profile inside the cone is also measured by the $\mathrm{x}$-ray image. Figure 3 shows the time-integrated $\mathrm{x}$-ray image together with neutron yield. The x-ray image in figure 3(a) indicates that the LFEX beam is focused at the center of the cone but absorbed at 100-200 $\mu \mathrm{m}$ away from the cone tip. The neutron yield increased by a factor of 30 from that without heating. Ion temperatures are deduced from the neutron yield, fuel density and fuel mass as shown in figure $3(b)$. We found that the ion temperature strongly depends upon the laser pulse width. When the pulse width is shorter than $2 \mathrm{ps}$, the neutron yield and the ion temperature are higher. From the above experimental results it can be seen that long-scale pre-plasmas are produced inside the cone and the main laser pulse is absorbed $100-200 \mu \mathrm{m}$ away from the cone tip. We presume that the LFEX laser pre-pulse produces the plasmas. Because of the long-scale pre-plasma by Kemp and Sentoku [6], a significant number of hot electrons with energy higher than $10 \mathrm{MeV}$ are generated in the underdense plasmas. These pre-pulse effects result in a decoupling between the hot electrons and the compressed core. Hence, the enhancement of the neutron yield is 30 times in comparison with the no-heating case and much smaller than the previous case [1].

\section{Pre-plasma inside cone}

The pre-plasma generated by a few $100 \mathrm{~mJ}$ pre-pulses with $10 \mathrm{~ns}$ in a high- $Z$ cone was simulated with the radiation hydrocode. The energy dependence, namely the intensity dependence, is shown in figure 4.

The pre-plasma density and the scale length increase with a decrease in the cone angle. In any case, the $10^{20} \mathrm{~cm}^{-3}$ density contours extend over $100 \mu \mathrm{m}$ when a $100 \mathrm{~mJ}$ pre-pulse is injected into the cone. Since such a pre-plasma is not good for realizing better coupling efficiency, as discussed in the previous section, the pre-pulse should be mitigated.

Two methods are possible for the mitigation of pre-pulses. One is using a plasma mirror and the other a thin foil. In the case of the plasma mirror, the distortion of phase front of the main pulse will be a problem, when the laser pulse duration is longer than 1 ps. The other method is using a thin foil at the entrance of the cone. The thin foil will cause a phase distortion 


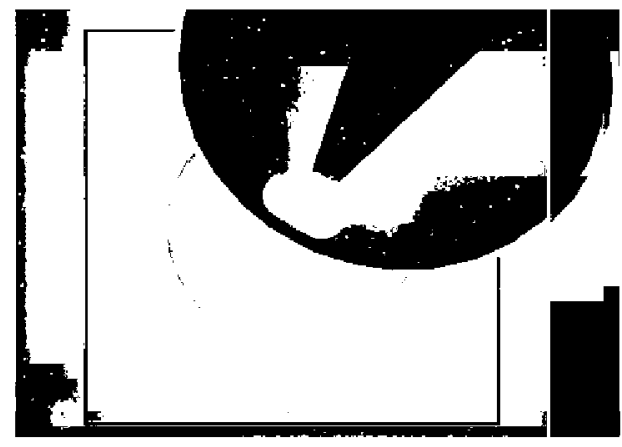

(a)

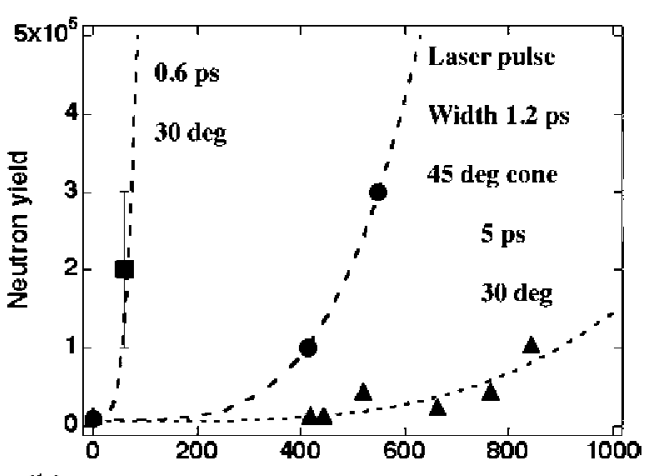

(b)

Heating laser energy (J)

Figure 3. (a) X-ray pinhole image of the plasma inside the cone and the imploded plasma, (b) neutron yields for the 2001 experiment, FIREX experiments with 5 and 2 ps heating pulse.
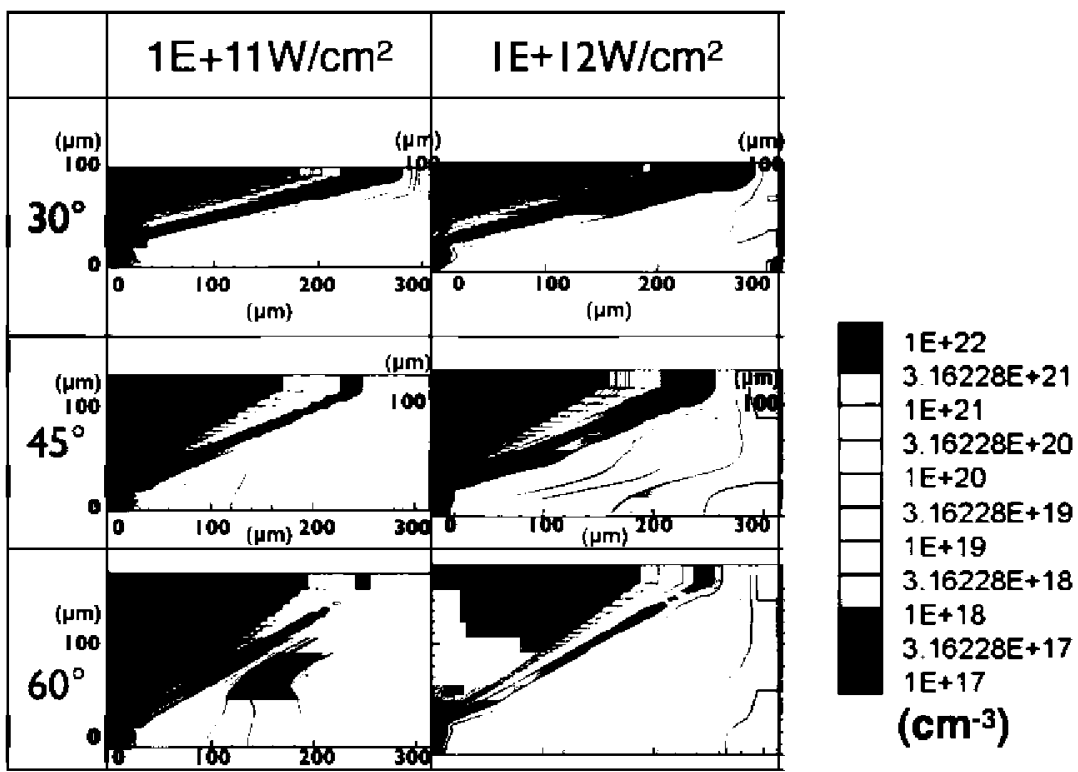

Figure 4. Pre-plasma profiles for cone angles of $30^{\circ}, 45^{\circ}$ and $60^{\circ}$ with $10^{11}$ and $10^{11} \mathrm{~W} \mathrm{~cm}^{-2}$. Here, a pre-pulse duration of $3 \mathrm{~ns}$ is assumed.

and laser absorption. However, it is applicable for any main pulse duration. The requirements on the foil thickness from the points of phase distortion and absorption are evaluated as follows.

We cover the entrance of the cone with a thin foil to mitigate the pre-pulse. The time dependences of the thin foil expansion are shown in the figure 5. The thickness of the foil is chosen to be enough to absorb the pre-pulse and to be thin enough not to absorb the main pulse.

According to the simulation in figure 5, this foil absorbs the pre-pulse sufficiently to reduce the pre-pulse level by a factor of 100 . When the average plasma density is less than $0.3 \%$ of the cut-off density as shown in figure 5 , the foil plasma may not disturb the phase front of the main pulse. The maximum phase distortion in the foil is evaluated by $L(1-n)$, which should be less 

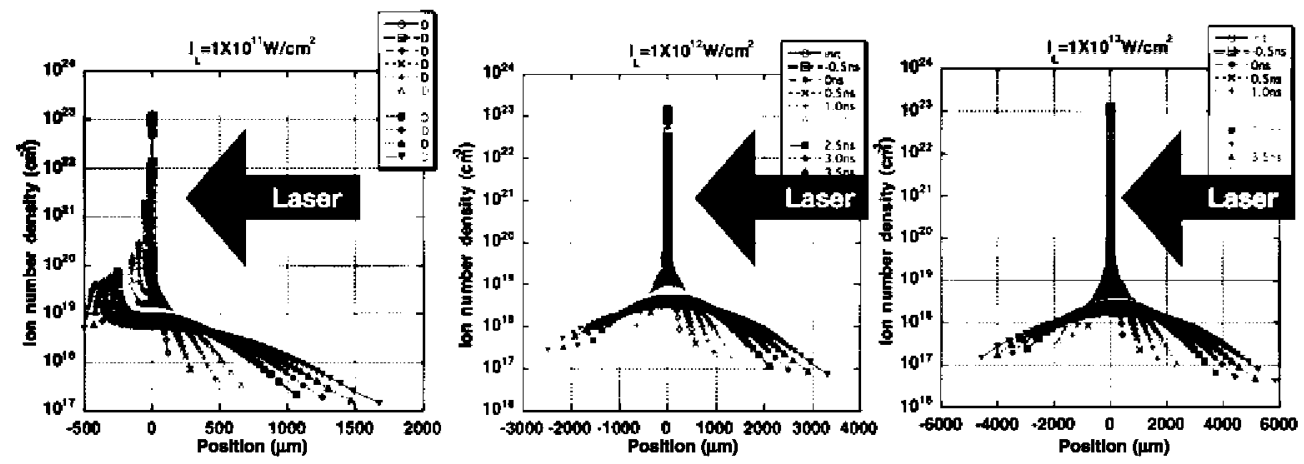

Figure 5. $0.1 \mu \mathrm{m}$ thick $\mathrm{CH}$ foil is irradiated with $4 \mathrm{~ns}$ pulse. The intensities are $10^{11}, 10^{12}$ and $10^{13} \mathrm{~W} \mathrm{~cm}^{-2}$.
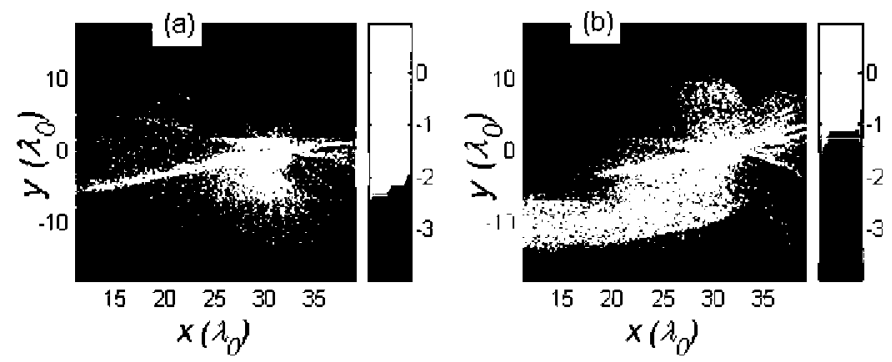

Figure 6. The electron energy density for single cone $(a)$ and double cone $(b)$ at time $t=1500 \mathrm{fs}$. Here the electron energy density is normalized by $m n_{\mathrm{cr}} c^{2}$.
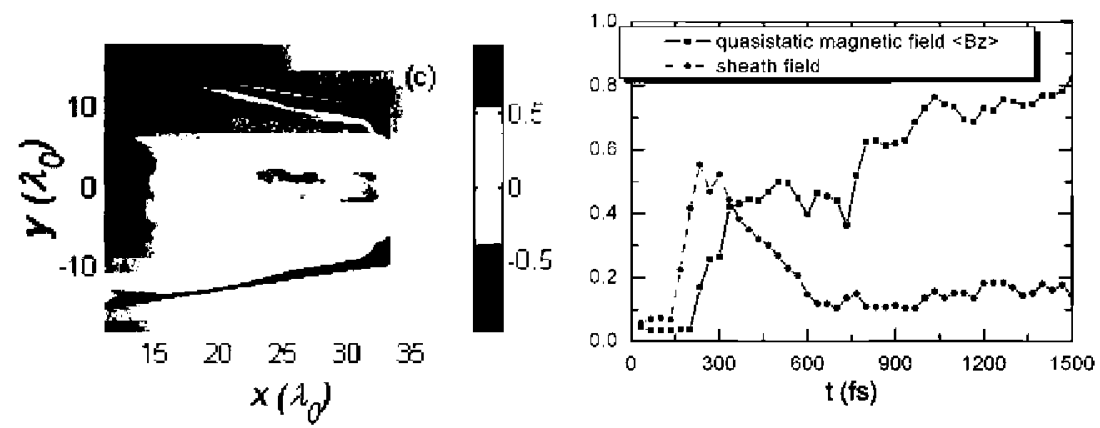

Figure 7. Electromagnetic field in a double cone: $(a)$ magnetic field structure at $1.5 \mathrm{ps},(b)$ time dependences of $E$ - and $B$-field.

than one laser wavelength. Here, $L$ is the effective plasma width which is about 2000 times laser wavelength and $n$ is the refractive index of the plasma which is given by $\left(1-n / \gamma n_{\mathrm{c}}\right)^{1 / 2}$. According to figure $5, n / n_{\mathrm{c}} \sim 1 / 300$. The main pulse laser intensity at the foil position may be one-tenth of the intensity at the cone tip, namely $10^{19} \mathrm{~W} \mathrm{~cm}^{-2}$. Hence, $\gamma$ is 3 . Therefore, the maximum phase shift will be one wavelength. This will be marginal. Therefore, the thickness of the thin foil is required to be smaller than $0.1 \mu \mathrm{m}$. As for the energy dissipation of the main pulse, it is assumed that the electrons of the foil are accelerated to $m c^{2}\left(\left(1+a^{2}\right)^{1 / 2}-1\right)$, where $a^{2}=\left(I_{\mathrm{L}} / 2.4 \times 10^{18} \mathrm{~W} \mathrm{~cm}^{2}\right)$ for $1 \mu \mathrm{m}$ laser. Then, the energy loss in the $0.1 \mu \mathrm{m}$ foil will be 
$2.8 \times 10^{5} \mathrm{~J} \mathrm{~cm}^{-2}$. Therefore, the diameter of the $300 \mu \mathrm{m}$ foil will absorb an energy of $200 \mathrm{~J}$. This also indicates that $0.1 \mu \mathrm{m}$ thickness is marginal.

\section{Double cone}

For improving the coupling efficiency, a new target, a so-called double cone target, has been proposed. Particle-in-cell simulations aimed at improving the coupling efficiency of the input laser energy deposited onto the compressed core using a double cone are carried out. It is found that high-energy electrons are effectively blocked by the vacuum gap inside the wing of the double cone (see figure 6).

Two main mechanisms to confine high-energy electrons are found, which are the sheath electric field at the rear of the inner cone wing and the quasi-static magnetic field inside the vacuum gap. However, the electrostatic retention of the high-energy electrons terminates within a few hundred femtoseconds as the vacuum gap is filled with the plasma. The quasi-static magnetic fields, though arise slowly in comparison with the electrostatic fields, but continue to grow to the order of $100 \mathrm{MG}$ (figure 7 ). The generation mechanism of the quasi-static magnetic fields is discussed in detail, and it is concluded that the quasi-static magnetic fields continue to confine the high-energy electrons for longer than a few picoseconds. The double cones result in the confinement and focusing of $22.3 \%$ of the input energy for deposition in the compressed core [7].

\section{Concluding remarks}

The multi-beam LFEX laser will be completed by April 2010 and the full beam LFEX experiment will start from the middle of 2010. The imploded dense plasma is expected to be heated up to the ignition temperature in 2011 [8]. In the forthcoming experiments, the advanced target shown in figure 2 will be used. The heating laser will be the two beams of the LFEX and $2 \mathrm{~kJ} \mathrm{ps}^{-1}$ laser energy will be injected. The ion temperature will reach $2 \mathrm{keV}$. This will be an important milestone toward realizing the $5 \mathrm{keV}$ ignition temperature.

\section{References}

[1] Kodama R et al 2001 Fast heating of super solid density matter as a step toward laser fusion ignition Nature $\mathbf{4 1 2} 798$

[2] Mima K et al 2007 Recent results and future prospects of laser fusion research at ILE, Osaka Eur. Phys. J. D $44259-64$

[3] Azechi H et al 2009 Nucl. Fusion 49104024

[4] Nagatomo H, Johzaki T, Nakamura T, Sakagami H, Sunahara A and Mima K 2007 Simulation and design study of cryogenic cone shell target for fast ignition FIREX project Phys. Plasmas 14056303

[5] Johzaki T et al 2008 Phys. Plasmas 15062702

[6] Kemp A and Sentoku Y 2009 Phys. Rev. E 79066406

[7] Cai H et al 2009 Phys. Rev. Lett. 102245001

[8] Mima K 2010 Inertial fusion power development: path for global warming suppression Nucl. Fusion 50014006 